\title{
Des curricula au cœur des défis
}

Études de cas : Chine (dont Shanghai), Inde, Japon, Singapour

Curricula at the core of challenges. Case studies: China (including Shanghai), India, Japan, Singapore

Los currículos en el centro de los retos. Estudios de casos : China (Shanghai incluida), la India, Japón, Singapur

\section{Roger-François Gauthier}

\section{(2) OpenEdition Journals}

Édition électronique

URL : https://journals.openedition.org/ries/4361

DOI : 10.4000/ries.4361

ISSN : 2261-4265

\section{Éditeur}

France Education international

Édition imprimée

Date de publication : 4 avril 2015

Pagination : 103-110

ISBN : 9782854206067

ISSN : $1254-4590$

\section{Référence électronique}

Roger-François Gauthier, «Des curricula au cœur des défis », Revue internationale d'éducation de Sèvres [En ligne], 68 I avril 2015, mis en ligne le 04 avril 2017, consulté le 03 juillet 2021. URL : http:// journals.openedition.org/ries/4361; DOI : https://doi.org/10.4000/ries.4361 


\title{
Des curricula au cœur des défis
}

\section{Études de cas : Chine (dont Shanghai), Inde, Japon, Singapour}

\author{
Roger-François Gauthier \\ Université Paris-Descartes, France
}

Les pays d'Asie ont pour beaucoup ceci de différent, si on les rapporte à d'autres régions extra-européennes, qu'ils ont depuis longtemps construit, souvent sous l'influence de doctrines fortes, comme le confucianisme dans la sphère des pays sinisés, des paysages d'une cohérence impressionnante en matière d'éducation publique. En commun en revanche avec d'autres régions du monde, ces pays ont ce fait que, pour divers motifs selon les cas (colonisations, guerres, influences diverses), ils se sont pour la plupart frottés, dans l'histoire, à des traditions éducatives européennes. Avec, de temps à autre, une infériorisation vis-à-vis de ces modèles, en miroir d'un regard historiquement critique de l'Occident sur les régimes scolaires de ces pays.

Or voici, tout à coup, que ces pays qui ont pour beaucoup une économie en plein essor, sont souvent cités, pour certains d'entre eux, en haut du peloton dans les comparaisons internationales en éducation...

Celui qui était facilement regardé comme archaïque apparaît peut-être à la pointe de la modernité, le copieur devient le copié (voir par exemple la fascination anglaise pour Singapour), et la tradition est interrogée sous forme d'un inventaire critique. Le choc Asie-Occident n'en finit plus de produire des ondes, mais désormais dans l'autre sens : les curricula asiatiques, dans le contexte mondialisé, intéressent l'Occident lointain...

Interroger quatre pays aussi différents que l'Inde, Singapour, la Chine (avec une vue particulière sur la région de Shanghai) et le Japon sur leurs problématiques et leurs évolutions en matière de curriculum permet certes de découvrir des histoires et situations spécifiques mais aussi de mettre en évidence des tensions largement communes sur les questions abordées.

Cet article s'inspire notamment des communications présentées lors du colloque international ${ }^{1}$ sur l'éducation en Asie, organisé par le Centre international d'études pédagogiques (Cui et Zhu, 2014 ; Sarangapani, 2014 ; Tan, 2014 ; Tsuneyoshi, 2014) ${ }^{2}$. Après avoir mentionné l'intérêt pour les questions

1. Colloque « L'éducation en Asie en 2014 : quels enjeux mondiaux ? » organisé les 12, 13 et 14 juin 2014 au Centre international d'études pédagogiques (CIEP), à Sèvres, à l'occasion du $20^{\mathrm{e}}$ anniversaire de la Revue internationale d'éducation de Sèvres. Pour en savoir plus : http://ries.revues.org/3710 (NdIR)

2. Sauf mention contraire, toutes les citations concernant ces pays sont extraites de ces communications publiées sur le site de la revue sur le portail OpenEdition revues.org : http://ries.revues.org/3710 (NdIR) 
curriculaires qui transparaît dans chaque cas, nous présenterons d'abord la tension entre le recours à des traditions curriculaires, elles-mêmes plurielles, et l'introduction volontariste de références nouvelles, puis la tension entre une conception très politique et centralisée de mise en œuvre des curricula et une conception décentralisée laissant plus de place au choix des acteurs. Nous évoquons enfin deux défis qui semblent, au-dessus de ces tensions, s’imposer dans presque tous les cas : celui de la marchandisation de l'école d'une part, d'autre part celui des difficultés rencontrées quand les curricula sont confrontés à la difficile question du multiculturalisme.

\section{DES PAYS SENSIBLES À L'IMAGE QUE DONNE D'EUX LEUR CURRICULUM}

À remarquer d'abord que, pour les quatre pays retenus, les questions curriculaires non seulement ne sont pas marginales, mais sont l'objet de beaucoup d'attention: chaque fois, les politiques éducatives s'expriment au moins autant sous la forme de politiques dites "substantielles» (ce que l'école doit permettre aux enfants d'apprendre) que de politiques structurelles ou organisationnelles. La présentation des questions curriculaires est d'ailleurs traversée par une conscience assez vive de l'originalité de situations nationales, comme de la valorisation de certaines spécificités. Si aucun des participants n’a prétendu que son pays devait apparaître comme modèle en matière de curricula, tous ont non seulement montré l'importance des questions curriculaires dans les politiques éducatives de leurs pays mais ont montré leur souhait que des images qui circulent à l'international soient parfois modifiées :

- la Chine veut montrer qu'elle n'est pas immobile, avec sept vagues successives de réformes curriculaires, mais il est aussi intéressant de voir combien Shanghai met un point d'honneur à apparaître régulièrement comme un cas différent du reste de la Chine en matière curriculaire, et si l'on veut " en avance", par exemple avec l'intention suivante : «faire passer les élèves du statut de récepteurs de connaissances passifs à celui d'apprenants actifs, en mettant l'accent sur l'éthique, l'innovation, les capacités pratiques, les aptitudes liées à l'information et la technologie, l'apprentissage expérimental et le développement personnel de chaque élève ";

- Singapour ne souhaite pas de son côté qu'on l'enferme dans l'image, qui a notamment son origine dans le PISA, d'un paradis pédagogique, en montrant qu'il est toujours à la recherche d'un équilibre curriculaire; cet équilibre s'avère d'autant plus difficile à trouver que le modèle des curricula séparés de bonne heure pour les enfants sur la base de concours non seulement ne favorise pas la cohésion sociale, mais que l'afflux de migrants (35\% de la population) renforce la nécessité et l'urgence d'une école non excluante ; 
- le Japon met un point d'honneur à ne pas être enfermé dans une image se réduisant à l'« enfer des examens». Ainsi, Ryoko Tsuneyoshi (2014) exprime une impatience quant au fait que le regard étranger sur l'école dans ce pays porte trop souvent sur des objets partiels, comme les résultats en mathématiques, au détriment d'aspects qui lui semblent plus fondateurs, comme la qualité bienveillante vis-à-vis des enfants de l'école de base, ou l'aura internationale de certains aspects collaboratifs en matière de formation des maitres (les «Lesson Studies»);

- en Inde aussi, comme on le verra plus loin, la civilisation et l'histoire de ce pays ont contribué à produire un curriculum spécifique qui fait de son école un objet ayant conscience de sa spécificité.

\section{TENSIONS ENTRE CULTURES TRADITIONNELLES ET QUESTIONNEMENTS CONTEMPORAINS}

Il y a traditions et traditions, celles que l'École reconnaît dans son projet, et celles qu'elle rejette. Elles n'ont pas toutes la même ancienneté, et elles ont aussi une tendance à s'ajouter, voire à s'hybrider, «ce qui fait que de multiples idées peuvent coexister tout en étant également légitimes».

$\mathrm{Si}$, dans un État récent comme Singapour, la référence explicite au " passé » n'a pas place, en revanche la référence à la méritocratie, formalisée par le pouvoir politique depuis l'indépendance, tient lieu en ce pays d'idéologie scolaire de base, avec par exemple le cortège des examens nationaux à tous les stades de la scolarité, ainsi qu'avec un long consensus sur le fait que l'école non seulement produit en bout de chaîne de l'inégalité, mais doit même en son sein faire une part visible, par des filières, par des curricula différents dès le primaire, aux « différences de capacités » des élèves;

La tradition des examens en Chine, malgré des épisodes de remise en cause aussi complètes que sous l'idéologie marxiste ou pendant la Révolution culturelle, réapparaît dès 1976 , avec le rétablissement de l'examen d'entrée à l'université.

De la même façon, Tsuneyoshi (2014) insiste sur la concurrence de deux idées sur l'école, l'une et l'autre très ancrées dans la réalité sociale : s'il existe bien une culture traditionnelle des examens et de la compétition à tout prix, débouchant au niveau des lycées à "l'enfer des examens", et si la pratique des jukus (cours du soir payants) vient exacerber cette tendance, toutefois, tout aussi ancré dans les valeurs sociales et en opposition paradoxale, cohabite, pour les niveaux primaire et collège, un modèle d'école inclusive très éloigné des modèles compétitifs : cette école, essentiellement publique, s'adresse "au cœur et à l'esprit " des enfants, c'est celle "du quartier », face à laquelle les familles n'exercent pas de choix particulier. Elle pratique la bienveillance, l'apprentissage 
collaboratif (dans le curriculum officiel), tenant famille et enfants plutôt à l'abri de l'angoisse scolaire. En termes de contenus, l'accent est mis sur la communication, la collaboration, la stabilité émotionnelle et d'autres qualités de l'enfant considéré comme un tout. Débats à l'initiative des enfants, réunions quotidiennes, organisation d'événements scolaires, nettoyage des locaux et service des repas en petits groupes... : tout cela fait partie de la routine quotidienne des enfants dans l'ensemble du pays.

En Inde aussi, nous dit Saraganpani (2014), se joue un rapport complexe à des traditions : le système indigène, antérieur à la colonisation, a rapidement dominé l'école coloniale, par exemple avec le règne de l'apprentissage par cour. «Encore aujourd'hui, nous trouvons trace dans les écoles gouvernementales de la notion de gourou et des méthodes traditionnelles d'enseignement et d'apprentissage. "En certains cas, sous l'apparence de formes nouvelles, « les vieilles recettes de traitement séparé des élèves " continuent à peser de tout leur poids. Et les traditions, ce sont aussi bien celles qui sont inacceptables et que l'État ambitionne de supprimer, comme le système des castes, que des éléments venant de réformateurs, parfois liés aux mouvements pédagogiques européens, qui ont proposé des modèles scolaires originaux il y a plus d'un siècle. Comme Swami Vivekanand, qui « a défini une vision de l'éducation destinée à forger la personnalité et la confiance en soi en s'inspirant la philosophie et de la pratique indigène des Védas ». Comme le poète Tagore proposant l'idée d'éducation par l'art et la Nature, en réagissant à la nature aliénante de l'éducation coloniale. Il faut citer aussi Jyotirao Phule, qui mettait l'accent sur «l'éducation des dalits (les intouchables) et des femmes et qui militait pour une éducation plus en phase avec les contextes ruraux. » Mais surtout Gandhi prônant par l'école un modèle économique tourné vers l'artisanat, une intégration de l'individu au monde et une lutte contre l'aliénation :

[...] une éducation qui encourage l'affirmation de soi, en lien avec toute une variété de modes de vie et de métiers traditionnels, et non pas orientée vers des emplois gouvernementaux ; (...) une éducation exaltant la confiance en soi et l'affirmation de la personnalité plutôt que la servilité, ouverte à tous, seul vecteur par lequel mener à bien les réformes sociales.

Si donc les héritages sont là, plus ou moins contestés, chaque pays est conduit aussi à s'en démarquer pour répondre à diverses préoccupations.

Il y eut par exemple en Inde, l'implantation, un temps, sous influence internationale, de la pédagogie par objectifs, qui émergea dans la première période qui suivit l'Indépendance, ou dès les années 1960 toute l'insistance mise sur le développement de l'enseignement scientifique, d'abord en matière de sciences dures, puis de sciences sociales. Ce furent aussi les thèmes et les préoccupations éducatives progressistes et centrées sur l'enfant, voire de pédagogies plus ludiques, qui ont régulièrement gagné du terrain, en même temps que multipliaient des critiques à l'égard d'un enseignement trop centré sur la mémoire. 
Au Japon, loin d'encourager la compétition éducative héritée, le débat national s'est concentré sur la manière de l'atténuer. On considérait que le pendule "était allé trop loin et qu'il fallait faire quelque chose pour desserrer l'étau de cette compétition à outrance ». Toutefois, signes des tensions, immédiatement après, certains constatent la baisse des performances scolaires, des études montreraient que les élèves des lycées japonais étudiaient moins que leurs camarades des autres pays, et que réussir les examens d'entrée n'était plus un facteur décisif de motivation. On fait aussitôt une marche arrière partielle : un pays comme le Japon estime que son rôle dans la "société de la connaissance ", à échelle mondiale, demande la vigilance, et le gouvernement, les entreprises et les médias insistent de plus en plus sur la nécessité de former des «compétences du niveau mondial».

En Chine, la réforme de 2001 entend bien proposer de nouvelles valeurs éducatives : d'une perspective étroite de transmission des connaissances dans la classe à une perspective orientée vers la question de savoir comment apprendre à apprendre et à développer des attitudes positives; d'une perspective orientée vers des disciplines à une perspective orientée vers les besoins des établissements et des élèves, de contenus périmés aux savoirs nécessaires pour la vie, du par cœur à la résolution de problèmes, d'une évaluation préoccupée seulement de sélection à une évaluation préoccupée du progrès de tous. Shanghai, de son côté, a introduit des parts de choix dans un curriculum jugé trop monolithique, en distinguant matières obligatoires, matières facultatives et matières extrascolaires.

À Singapour, confronté aux limites, notamment en termes d'équité et de cohésion sociale, du système méritocratique pur, le gouvernement a tenté de gommer les frontières nettes séparant les différentes filières, et de proposer une grande variété de filières et de passerelles, affirmant que toutes les formes de réussite se valaient...

\section{TENSIONS ENTRE \\ CENTRALISATION POLITIQUE ET MISE EN GUVRE \\ PAR LES ACTEURS LOCAUX}

La question du rôle du pouvoir politique en matière curriculaire montre une tendance centralisatrice qui a dominé dans l'histoire récente. Ce fut le cas notamment, dans un contexte post colonial tel que celui de Singapour, où il s'agissait, à l'indépendance (1965), de surmonter un héritage fait "d'un fatras disparate d'établissements fonctionnant dans quatre langues différentes: l'anglais, le chinois, le malais et le tamoul » et de prendre en mains la question scolaire : "la scolarisation était loin d'être universelle et les établissements manquaient d'objectifs communs, voire même de programmes ou d'examens communs». 
À l'indépendance, l'école singapourienne a été chargée d'une fonction politique forte, et l'État a constitué un système unifié pour instaurer un ordre éducatif face au désordre préexistant. Les deux objectifs fixés à l'école étaient et sont fortement demeurés le fait de favoriser aussi bien la croissance économique que la cohésion sociale. L'enseignement privé n'avait logiquement dans ce contexte qu'une faible part et a vu son influence réduite.

L'Inde a hérité d'une centralisation éducative d'origine coloniale, ou liée aux anciens royaumes, qui se marque toujours fortement malgré la diversité des évolutions et influences. Cette centralisation a bien eu sûr comme thème privilégié, dont s'empare l'État, le développement d'une école contre le système des castes. À noter enfin que, dans les difficultés de développer l'école pour tous, la nécessité de répartir l'aide internationale a été un nouveau facteur de centralisation. L'interaction avec la politique ne met par ailleurs pas à l'abri d'une valorisation du contexte indien et, aussi, du nationalisme hindou.

La Chine est naturellement très symptomatique d'une situation politique changeante au niveau du pouvoir central qui, depuis le premier curriculum national, s'est reflétée dans des changements curriculaires réguliers. Même dans des cas où s'amorçait une certaine décentralisation, comme vers Shanghai, on peut dire que « la délégation de la mise au point du curriculum du gouvernement central aux autorités locales a créé de fait une nouvelle centralisation, aux mains des autorités éducatives de Shanghai ».

On voit toutefois que les pays tendent souvent à faire une plus grande place à une décentralisation curriculaire, qui va de pair avec une plus grande prise en compte des réalités géographiques et sociales, mais aussi avec le souci de faire plus de place au professionnalisme d'enseignants davantage définis alors comme des innovateurs que comme des exécutants.

C'est le cas de Singapour, où l'autorisation d'une offre plus différenciée apparaît comme une réponse à une diversité effective de contextes d'enseignement. On recherche un équilibre entre la centralisation des décisions politiques en matière d'éducation et la délégation des prises de décision aux chefs d'établissement.

Ce fut surtout le cas en Chine, où les réformes ont aussi consisté à dépasser un modèle initial centralisé : plus d'écoute et moins d'injonctions, mise en commun de ressources décentralisées, part plus importante laissée au curriculum local, approches innovantes en formation des enseignants, appel à leur réflexion innovante. Différents développements informatiques, comme le site New Century Curriculum Network (créé par le Centre du curriculum de l'enseignement fondamental du ministère de l'Éducation), permettent la mise en commun de ressources humaines de qualité et les rendent disponibles en ligne, en passant par des canaux officiels. Il faudrait citer aussi le Big Name Teacher Studio (géré par d'excellents enseignants) " qui fournit tout un éventail de savoirs et de pratiques». On a aussi introduit de nouvelles techniques de direction et 
des approches managériales fondées sur la culture occidentale, qui confrontent les chefs d'établissement à de véritables dilemmes culturels (en ce qui concerne par exemple la direction par délégation ou management du zéro défaut).

\section{MARCHANDISATION ET MULTICULTUREL : LES DEUX DÉFIS POSÉS AUX POLITIQUES CURRICULAIRES}

Ces évolutions, qui font leur chemin entre valeurs traditionnelles et « modernisations » diverses, entre dirigisme politique et décentralisations plus ou moins régulées, se heurtent toutefois à deux défis délicats.

La marchandisation et tous les phénomènes qu'elle amène imposent certains jeux aux responsables. La compétition qu'ils essaient parfois de limiter à l'interne réapparaît à l'international. Si, par exemple, devant la montée en puissance des inégalités de revenus, le gouvernement singapourien a tenté, au cours des dix dernières années, de remplacer les filières par la répartition en groupes de niveaux dans le primaire, et d'atténuer les différences entre filières dans le secondaire, la marchandisation, qui propose une offre (par exemple en multipliant les établissements concurrents) qui satisfait les riches, exacerbe les inégalités sociales, qui s'ajoutent aux inégalités ethniques.

Des questions comme l'évaluation du curriculum ou le recrutement des enseignants sont de plus en plus aux mains d'entreprises privées. Le lobbysme de nouveaux " maîtres à penser ", jouant de leur influence auprès de la bureaucratie et cherchant à mettre la main sur l'espace politique trouble, par exemple en Inde, le travail sur le curriculum dont la puissance publique se sent investi.

On peut même dire qu'au Japon, c'est tout l'équilibre d'une éducation dans laquelle se reconnaissaient les classes moyennes qui tend à disparaître, puisque, selon leur fortune, les familles font appel à des écoles privées présentant des curricula différents.

Ces pays sont confrontés aussi, dans leurs développements curriculaires, à des variations importantes dans l'identité culturelle des élèves. C'est bien sûr le cas dans un petit pays comme Singapour, où l'immigration a pour conséquence que les non-citoyens représentent $37 \%$ des 5 millions d'habitants. Déjà les différentes communautés ethniques ont des taux de succès très différents à l'école : au cours des trente dernières années, le gouvernement a apporté son aide aux groupes de soutien internes aux différentes communautés ethniques afin d'améliorer leur réussite scolaire, avec des succès limités. Le gouvernement a réagi avec vigueur par la mise en place en 2011 de la révision du curriculum portant sur l'éducation à la personnalité et à la citoyenneté, qui a élargi le concept de citoyenneté pour inclure les compétences interculturelles. Des besoins similaires d'ouverture au multiculturel existent au Japon, ouverture qui est sans doute un trait plus traditionnel de l'éducation indienne, habituée à considérer des 
identités locales, des savoirs et des cultures non occidentales. L'Inde est toutefois, quant à elle, confrontée à une présence de plus en plus grande de la religion dans le curriculum, ainsi qu'à un développement du nationalisme hindou.

Mais Shanghai a de la même façon à résoudre un défi quelque peu similaire. Il s'agit de :

[...] combler le large fossé qui sépare les habitants du centre-ville de ceux des banlieues, les élèves nés à Shanghai de ceux qui viennent de s'y installer, comme il conviendrait de réduire les différences entre établissements provoquées par les traditions historiques et les qualités professionnelles des enseignants qui y exercent. (Xu, 2012, cité dans Cui et Zhu, 2014)

Peut-être pourrait-on considérer que ces curricula sont d'un côté tentés de rechercher des standards mondialisés, en espérant ne pas y perdre leur âme, en quête d'une éducation dont le périmètre ne serait plus ou plus seulement celui de l'État, mais qu'à l'autre extrémité, l'écoute des élèves et de leurs parents, dans leur diversité, est un continent encore peu abordé ?

\section{BIBLIOGRAPHIE}

CUI Y. et ZHU Y. (2014) : "Les réformes curriculaires en Chine, hier et aujourd'hui », Revue internationale d'éducation de Sèvres, [en ligne], colloque "L'éducation en Asie en 2014 : Quels enjeux mondiaux? ", mis en ligne le 5 juin 2014. http://ries.revues. org/3841/; version anglaise : http://ries.revues.org/3846/

SARANGAPANI P.M. (2014) : "Savoir, curricula et méthodes pédagogiques : le cas de l'Inde ", Revue internationale d'éducation de Sèvres, [en ligne], colloque "L'éducation en Asie en 2014 : Quels enjeux mondiaux? », mis en ligne le 5 juin 2014. http://ries. revues.org/3848/; version anglaise : http://ries.revues.org/3851/

TAN J. (2014) : «L'enseignement à Singapour : pour quoi ? Pour qui ? Revue internationale d'éducation de Sèvres, [en ligne], colloque "L'éducation en Asie en 2014 : Quels enjeux mondiaux? ", mis en ligne le 5 juin 2014. http://ries.revues.org/3852/; version anglaise : http://ries.revues.org/3856/

TSUNEYOSHI R. (2014) : "Des modèles de scolarisation à l'ère mondiale : le cas du Japon ", Revue internationale d'éducation de Sèvres, [en ligne], colloque "L'éducation en Asie en 2014 : Quels enjeux mondiaux?», mis en ligne le 5 juin 2014. http://ries. revues.org/3857/; version anglaise : http://ries.revues.org/3857/ 